\title{
A golden ticket to the brain
}

Gene editing technologies such as the CRISPR system are at the forefront of new treatment strategies for genetic diseases because they enable the precise and permanent editing of DNA in a living organism. We can also apply CRISPR in the brain but mainly by using viruses as delivery vehicles - an approach that is not amenable to the clinic. Now, writing in Nature Biomedical Engineering, Hye Young Lee, Niren Murthy and colleagues report the delivery of the CRISPR system to specific regions of the brain using gold nanoparticles to modify gene expression in brain cells for the treatment of autism-related fragile $\mathrm{X}$ syndrome in mice.

Many neurological diseases are caused by genetic mutations, overexpression or increased activation of specific genes. The CRISPR-associated protein 9 (Cas9) and CRISPR-associated endonuclease in Prevotella and Francisella 1 (Cpf1) are RNA-guided DNA endonucleases. Using a virus to deliver these is a promising technique to achieve

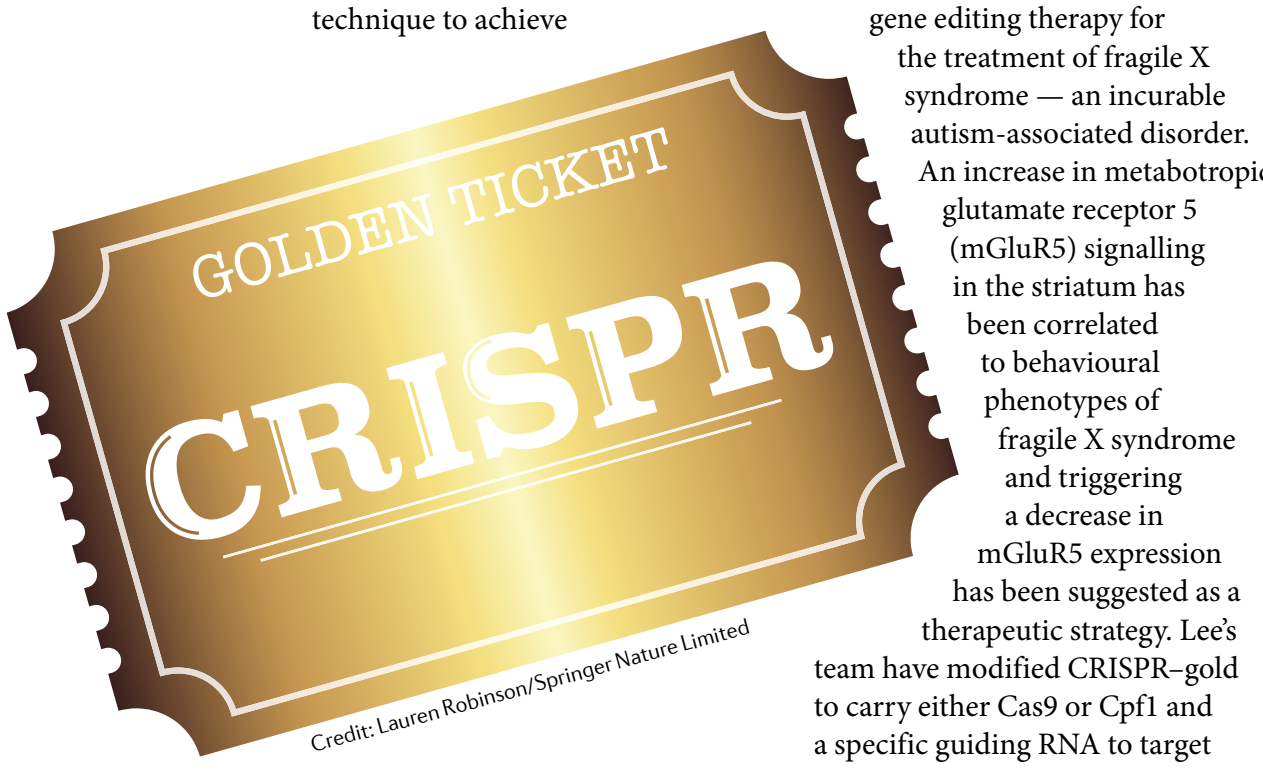

permanent knock-down or correction of genes, but comes with safety concerns associated with the inherent immunogenicity of viruses and the genomic damage caused by prolonged expression of the enzymes.

An alternative approach makes use of CRISPR-gold, a non-viral delivery vehicle featuring gold nanoparticles conjugated with thiol-modified single-stranded DNA. These strands hybridize with donor DNA that binds Cas9 or Cpf1 proteins with a pendant guiding RNA that targets a specific DNA sequence. The modified nanoparticles can then be encapsulated with an endosomal disruptive polymer to enable internalization by cells through endocytosis. "Delivering the CRISPR system into the brain without using a virus greatly increases the potential for clinical translation of gene editing as a treatment for brain disorders," explains Lee.

Lee and colleagues have applied the CRISPR-gold technology to develop a CRISPR-based, localized ene editing therapy for the treatment of fragile $\mathrm{X}$ syndrome - an incurable autism-associated disorder glutamate receptor 5 (mGluR5) signalling in the striatum has fragile X syndrome nd triggering mGluR5 expression has been suggested as a eam have modified CRISPR-gold a specific guiding RNA to target and knock-down mGluR5. "The modified gold nanoparticles can be directly delivered into the striatum of the brain through intracranial injection," explains Lee. "Using this approach, we achieve a $40-50 \%$ reduction in mGluR5 protein levels in mice, without any observable off-target effects." Importantly, this decrease in protein expression is confined to the striatum and translates into the behavioural rescue of mice with autistic phenotypes, for example, less exaggerated repetitive behaviour.

The knock-down of genes by CRISPR-gold is permanent and treatment involves only a single injection. The researchers also demonstrated that CRISPR-gold can be used to edit genes in multiple brain cell types, including neurons, microglia and astrocytes cells that are difficult to edit using viral delivery.

Although the gold nanoparticle delivery strategy is biocompatible and non-toxic, questions remain regarding the clearance and elimination of the gold cores from cells. "We are currently investigating different nanomaterials for the delivery of CRISPR into the brain," says Lee. "This is especially important for clinical translation to avoid any possible toxic side effects of the remaining gold cores." The team is also working on a cell-type-specific version of CRISPR-gold using cellspecific markers and is exploring the method for the treatment of other brain disorders, such as epilepsy and brain tumours.

Christine-Maria Horejs

ORIGINAL ARTICLE Lee, B. et al. Nanoparticle delivery of CRISPR into the brain rescues a mouse model of fragile $X$ syndrome from exaggerated repetitive behaviours. Nat. Biomed. Eng. https:// doi.org/10.1038/s41551-018-0252-8 (2018) 